\title{
STEADY STATES OF A SYSTEM OF PARTIAL DIFFERENTIAL EQUATIONS MODELING MICROBIAL ECOLOGY*
}

\author{
SZE-BI HSU ${ }^{\dagger}$
}

Abstract. In this paper we discuss the existence and uniqueness of solutions for the boundary value problem

$$
\begin{aligned}
& u^{\prime \prime}(x)=F(u(x)) v(x), \\
& \lambda v^{\prime \prime}(x)=-[\kappa F(u(x))-\theta] v(x), \quad 0 \leq x \leq 1, \lambda, \kappa, \theta>0, \\
& u^{\prime}(0)=0, \quad u(1)=1, \\
& v^{\prime}(0)=0, \quad v^{\prime}(1)=0,
\end{aligned}
$$

which arises in microbial ecology. The growth rate $F(u)$ of bacteria satisfies $F(0)=0, F^{\prime}(u)>0$. We study this problem by using Rabinowitz's global bifurcation theorem and the maximum principle.

1. Introduction. In [1], D. Lauffenburger, R. Aris and K. Keller study the effects of random motility on growth of bacterial populations. Consider a population of bacterial cells confined to a finite region, with a diffusible chemical substrate present in the medium. This substrate is assumed to be the nutrient that is rate limiting for growth, and it is further assumed that it enters the region at a boundary. For simplicity, we consider one-dimensional geometry, with uniform conditions in the transverse dimensions, so that the cells are confined to the region $0 \leq x \leq L$. Substrate enters the region at the boundary $x=L$, and is present there at a constant concentration determined by ambient conditions. We assume Monod's model for the growth of bacterial populations along with exponential nonviability or death. Then the model equations are

$$
\begin{aligned}
& \frac{\partial b}{\partial t}=\mu \frac{\partial^{2} b}{\partial x^{2}}+\left[f(s)-k_{e}\right] b, \\
& \frac{\partial s}{\partial t}=D \frac{\partial^{2} s}{\partial x^{2}}-\frac{1}{Y} f(x) b
\end{aligned}
$$

for $0 \leq x \leq L$. The boundary conditions are

$$
\begin{array}{lll}
\frac{\partial b}{\partial x}=0, & s=s_{0} & \text { at } x=L, \\
\frac{\partial b}{\partial x}=0, & \frac{\partial s}{\partial x}=0 & \text { at } x=0 .
\end{array}
$$

Here:

$$
f(s)=m s /(K+s) \text {, }
$$

$b(x, t)=$ bacterial cell density at position $x$ and time $t$ (mass of viable cells per volume of medium),

$s(x, t)=$ substrate concentration at position $x$ and time $t$ (mole of substrate per volume of medium),

$\mu=$ random motility coefficient of bacterial cells,

$D=$ substrate diffusion coefficient,

${ }^{*}$ Received by the editors July 9,1982 . This research was partially supported by the National Science Council of the Republic of China.

${ }^{\dagger}$ Department of Applied Mathematics, Chiao-Tung University, Hsin-Chu, Taiwan. 
$k_{e}=$ death rate of bacteria population,

$Y=$ yield coefficient (mass of viable cells produced per mole of substrate),

$s_{0}=$ constant concentration of substrate present at boundary $x=L$,

$m=$ maximal growth rate of bacterial cells,

$K=$ the half-saturation constant.

Introducing new dimensionless parameters

$$
\begin{aligned}
& u=\frac{s}{s^{(0)}}, \quad \xi=\frac{x}{L}, \quad \tau=\frac{D t}{L^{2}}, \quad v=\frac{b m L^{2}}{Y s^{(0)} D}, \quad \theta=\frac{L^{2}}{D} k_{e}, \quad \lambda=\frac{\mu}{D}, \\
& \kappa=\frac{L^{2}}{D} m, \quad F(u)=\frac{1}{m} f\left(s^{(0)} u\right)=\frac{u}{K / s^{(0)}+u}
\end{aligned}
$$

yields equations

$$
\frac{\partial u}{\partial \tau}=\frac{\partial^{2} u}{\partial \xi^{2}}-F(u) v, \quad \frac{\partial v}{\partial \tau}=\lambda \frac{\partial^{2} v}{\partial \xi^{2}}+(\kappa F(u)-\theta) v
$$

with boundary conditions

$$
\begin{array}{ll}
u(1, \tau)=1, & \frac{\partial u}{\partial \xi}(0, \tau)=0 \\
\frac{\partial v}{\partial \xi}(1, \tau)=0, & \frac{\partial v}{\partial \xi}(0, \tau)=0 .
\end{array}
$$

In [1] the authors assume $F(u)=1$ for $u>u_{c}$ and 0 for $u \leq u_{c}$, where $F\left(u_{c}\right)=\theta / \kappa$, and compute the steady states of (1.3), (1.4). That is, they try to solve the nonlinear problem (1.3), (1.4) by linear techniques. The main purpose of this paper is to show the existence and uniqueness of steady states of (1.3), (1.4). Our technique is to apply the global bifurcation theorem of Rabinowitz [5] and the maximum principle [6].

2. Statements of main results. Consider the steady state problems of (1.3), (1.4)

$$
\begin{aligned}
& u^{\prime \prime}(x)=F(u(x)) v(x) \\
& v^{\prime \prime}(x)=-(\kappa F(u(x))-\theta) v(x)
\end{aligned}
$$

for $0 \leq x \leq 1$ with boundary condition

$$
\begin{array}{ll}
u(1)=1, & u^{\prime}(0)=0, \\
v^{\prime}(1)=0, & v^{\prime}(0)=0 .
\end{array}
$$

We may assume that $F(u)$ satisfies

$$
F(0)=0, \quad F^{\prime}(u)>0 \quad \text { for } u>0 .
$$

Our main result is the following theorem.

THEOREM 2.1. (i) If $\kappa F(1)-\theta<0$ then the trivial solution $\left(u_{0}(x), v_{0}(x)\right)$ of $(2.1)$, (2.2) is the unique nonnegative solution where $u_{0}(x) \equiv 1, v_{0}(x) \equiv 0$. 
(ii) If $\kappa F(1)-\theta>0$, then there exists a unique solution $(u(x), v(x))$ of $(2.1),(2.2)$ with $u(x)>0, v(x)>0$ for $0 \leq x \leq 1$.

3. Proof. Our approach is very similar to that of Cushing [2] and Butler et al. [3]. Before we prove our main theorem, we note the following lemmas.

LEMMA 3.1. Let $(u(x), v(x))$ be a solution of (2.1) and (2.2) with $u(x) \geq 0, v(x) \geq 0$, $0 \leq x \leq 1$. Then

(i) $0 \leq u(x) \leq 1$.

(ii) If $(u, v) \equiv\left(u_{0}, v_{0}\right)$ and $\kappa F(1)-\theta>0$, then $u(x)$ is a strictly convex and strictly increasing function on $0 \leq x \leq 1$ while $v(x)$ is a strictly increasing function on $0 \leq x \leq 1$, and there exists $0<x_{0}<1$ such that $v(x)$ is strictly convex on $\left(0, x_{0}\right)$ and strictly concave on $\left(x_{0}, 1\right)$.

Proof. From $u^{\prime}(0)=0, u(1)=1$ and $u^{\prime \prime} \geq 0$ (i) follows easily. If $(u, v) \neq\left(u_{0}, v_{0}\right)$, then obviously $u(0) \neq 1$; otherwise $u \equiv 1$ and $v \equiv 0$. From the uniqueness of solutions of ODE's and the first equation of $(2.1), u(0) \neq 0$. Hence $u(x)>0$ for $0 \leq x \leq 1$. We claim $v(x)>0$ for $0 \leq x \leq 1$. From the uniqueness of solutions of ODE's and the second equation of $(2.1), v(0)>0$. Suppose the claim is not true. Then there exists $0<\xi<1$ such that $v(\xi)=0$ and $v^{\prime}(\xi)=0$. Then $v(x) \equiv 0$, and this is the desired contradiction. Hence $u^{\prime \prime}>0$ on $(0,1)$ and $u(x)$ is a strictly convex and strictly increasing function on $0 \leq x \leq 1$. Obviously it is impossible to have $\kappa F(u(x))-\theta>0$ for all $0 \leq x \leq 1$, since then $v^{\prime \prime}(x)<0$ for $0 \leq x \leq 1$, which contradicts to the boundary conditions $v^{\prime}(0)=0=v^{\prime}(1)$. Hence there exists a unique $x_{0}, 0<x_{0}<1$, such that $\kappa F\left(u\left(x_{0}\right)\right)-\theta=0$ and $v^{\prime \prime}(x)>0$ for $0<x<x_{0}, v^{\prime \prime}(x)<0$ for $x_{0}<x \leq 1$. Obviously $v(x)$ is strictly increasing on $[0,1]$.

Proof of Theorem 2.1(i). Suppose $(u(x), v(x))$ is a nonnegative steady state, $(u, v) \neq\left(u_{0}, v_{0}\right)$. Then $u(x) \neq 1$ and $v(x) \neq 0$. From the second equation of (2.1), boundary conditions $v^{\prime}(0)=v^{\prime}(1)=0$ and Lemma 3.1 (i), it follows that

$$
0=-\int_{0}^{1} v(x)[\kappa F(u(x))-\theta] d x>-\int_{0}^{1} v(x)[\kappa F(1)-\theta] d x>0 .
$$

This is a contradiction. Hence we complete the proof.

Before we prove the second part of Theorem 2.1, we need to state the local and global bifurcation theorems, respectively, due to Krasnoselskii [4] and Rabinowitz [5].

LEMMA 3.2 [4]. Let $T_{\lambda}=\lambda A+D$ be a continuous one-parameter family of operators from a Banach space $X$ to itself, such that $A$ is compact and linear and satisfies $\|D x-D y\|$ $=o(\|x-y\|)$. Then a bifurcation of the equation $T_{\lambda} x=x(x \in X)$ can only occur at characteristic value $\lambda^{*}$ (reciprocal of a nonzero eigenvalue) of $A$, and will occur if $\lambda^{*}$ has odd multiplicity. In this case, the bifurcation point corresponds to a continuous branch of eigenvectors of $T_{\lambda}$ in a neighborhood of the zero of $X$.

Lemma 3.3 [5]. Let $T_{\lambda}, A, D, X$ be as above, and let $S$ be the closure of the set of all nontrivial solutions of $T_{\lambda} x=x$ as $\lambda$ ranges over $\mathbb{R}$. If $\lambda^{*}$ is a simple characteristic value of $A$, then $S$ contains two subcontinua $C_{\infty}^{+}, C_{\infty}^{-}$whose only point in common for $\lambda$ near $\lambda^{*}$ is $\left(\lambda^{*}, 0\right)$, and each of which either

(a) is unbounded, or

(b) contains $(\hat{\lambda}, 0)$ where $\hat{\lambda} \neq \lambda^{*}$ is a characteristic value of $A$.

LEMMA 3.4. For any positive solution $(u, v)$ of $(2.1)$ we have

$$
v(0) \geq \frac{\kappa}{\lambda}\left[\frac{2((\lambda / \kappa) v(1)+1)}{e^{\sqrt{\alpha}}+e^{-\sqrt{\alpha}}}-u(0)\right] \text { where } \alpha=\frac{\theta}{\lambda} .
$$


Proof. From (2.1) we have the following inequality:

$$
\begin{aligned}
& u^{\prime \prime}+\frac{\lambda}{\kappa} v^{\prime \prime}=\frac{\theta}{\kappa} v<\alpha\left(u+\frac{\lambda}{\kappa} v\right), \quad \text { where } \alpha=\frac{\theta}{\lambda}, \\
& \left(u+\frac{\lambda}{\kappa} v\right)^{\prime}(0)=0, \\
& \left(u+\frac{\lambda}{\kappa} v\right)(1)=1+\frac{\lambda}{\kappa} v(1) .
\end{aligned}
$$

Comparing (3.1) with the equations

$$
\begin{aligned}
& U^{\prime \prime}=\alpha U, \\
& U^{\prime}(0)=0, \quad U(1)=1+\frac{\lambda}{\kappa} v(1)
\end{aligned}
$$

yields

$$
U(x)=\left(\frac{(\lambda / \kappa) v(1)+1}{e^{\sqrt{\alpha}}+e^{-\sqrt{\alpha}}}\right)\left(e^{\sqrt{\alpha} x}+e^{-\sqrt{\alpha} x}\right) \leq u(x)+\frac{\lambda}{\kappa} v(x), \quad 0 \leq x \leq 0 ;
$$

in particular,

$$
v(0) \geq \frac{\kappa}{\lambda}\left[\frac{2((\kappa / \lambda) v(1)+1)}{e^{\sqrt{\alpha}}+e^{-\sqrt{\alpha}}}-u(0)\right] \geq \frac{\kappa}{\lambda}\left[\frac{2((\lambda / \kappa) v(1)+1)}{e^{\sqrt{\alpha}}+e^{-\sqrt{\alpha}}}-1\right] .
$$

Proof of Theorem 2.1 (ii) (existence). Setting $U=u-u_{0}, V=v-v_{0}$ in (2.1), we have for $0 \leq x \leq 1$,

$$
\begin{aligned}
& U^{\prime \prime}=F(1) V+g_{1}(U, V), \\
& \lambda V^{\prime \prime}=\theta V-\kappa F(1) V+g_{2}(U, V), \\
& U(1)=0, \quad U^{\prime}(0)=0, \quad V^{\prime}(0)=0, \quad V^{\prime}(1)=0
\end{aligned}
$$

where $g_{1}(U, V)=o(\|(U, V)\|), g_{2}(U, V)=o(\|(U, V)\|)$ as $(U, V) \rightarrow(0,0)$. Consider the linear system

$$
\begin{aligned}
& U^{\prime \prime}=F(1) V, \\
& \lambda V^{\prime \prime}=\theta V, \quad 0 \leq x \leq 1, \\
& U(1)=0, \quad U^{\prime}(0)=0, \quad V^{\prime}(0)=0, \quad V^{\prime}(1)=0 .
\end{aligned}
$$

It is easy to show that (3.3) has only the trivial solution $U \equiv 0, V \equiv 0$. Let $B$ be the Banach space of continuous function on $0 \leq x \leq 1$ with the supremum norm. If $h_{1}$, $h_{2} \in B$, let $L_{1}\left(h_{1}\right), L_{2}\left(h_{2}\right)$ respectively, be the unique solutions of

$$
\begin{aligned}
& U^{\prime \prime}=h_{1}, \quad U^{\prime}(0)=0, \quad U(1)=0, \\
& \lambda V^{\prime \prime}=\theta V+h_{2}, \quad V^{\prime}(0)=0, \quad V^{\prime}(1)=0 .
\end{aligned}
$$

Obviously $L_{1}, L_{2}: B \rightarrow B$ are linear and compact operators.

Write (3.2) formally as the following operator equation:

$$
\left(\begin{array}{l}
U \\
V
\end{array}\right)=\kappa L^{*}\left(\begin{array}{l}
U \\
V
\end{array}\right)+G\left(\begin{array}{l}
U \\
V
\end{array}\right)
$$


where

$$
\begin{gathered}
L^{*}\left(\begin{array}{c}
U \\
V
\end{array}\right)=\left(\begin{array}{c}
-F(1) L_{1} \circ L_{2}(F(1) V) \\
L_{2}(-F(1) V)
\end{array}\right), \\
G\left(\begin{array}{c}
U \\
V
\end{array}\right)=\left(\begin{array}{c}
F(1) L_{1} \circ L_{2}\left(g_{2}(U, V)\right)+L_{1}\left(g_{1}(U, V)\right) \\
L_{2}\left(g_{2}(U, V)\right)
\end{array}\right)
\end{gathered}
$$

and $L^{*}: B \times B \rightarrow B \times B$ is compact and linear while $G: B \times B \rightarrow B \times B$ is compact and $G(U, V)=o(\|(U, V)\|)$ as $\|(U, V)\| \rightarrow 0$. We now formally treat $\kappa$ in (3.5) as a real parameter. Consider the eigenvalue problem

$$
\left(\begin{array}{l}
U \\
V
\end{array}\right)=\kappa L^{*}\left(\begin{array}{l}
U \\
V
\end{array}\right)
$$

ClaIM. The characteristic values of $L^{*}$ are $\kappa^{*}=\theta / F(1)$ and

$$
\kappa_{n}=\frac{\theta+\lambda(n \pi)^{2}}{F(1)}, \quad n=1,2, \cdots .
$$

Let $\kappa$ be a characteristic value of $L^{*}$. Then there exists $\left(\begin{array}{l}U \\ V\end{array}\right) \neq\left(\begin{array}{l}0 \\ 0\end{array}\right)$ such that

$$
\left(\begin{array}{l}
U \\
V
\end{array}\right)=\kappa L^{*}\left(\begin{array}{l}
U \\
V
\end{array}\right)=\kappa\left(\begin{array}{c}
-F(1) L_{1} \circ L_{2}(F(1) V) \\
L_{2}(-F(1) V)
\end{array}\right)
$$

or the system

$$
\begin{aligned}
& U^{\prime \prime}=F(1) V, \\
& \lambda V^{\prime \prime}=\theta V-\kappa F(1) V, \\
& U(1)=0, \quad U^{\prime}(0)=0, \quad V^{\prime}(0)=0, \quad V^{\prime}(1)=0
\end{aligned}
$$

has nontrivial solutions.

If $\kappa F(1)-\theta<0$ then $U \equiv 0, V \equiv 0$.

If $\kappa F(1)-\theta=0$ then the eigenspace belonging to $\left(\kappa^{*}\right)^{-1}=(\theta / F(1))^{-1}$ is generated by $\left(U_{1}, V_{1}\right)$, where $U_{1}(x)=(F(1) / 2)\left(x^{2}-1\right)$ and $V_{1}(x) \equiv 1$. If $\kappa F(1)-\theta>0$ then $V^{\prime \prime}+$ $\alpha V=0, \alpha=(\kappa F(1)-\theta) / \lambda>0, V^{\prime}(0)=0=V^{\prime}(1)$.

In order to have $V Z 0, \alpha$ must satisfy $\sqrt{\alpha}=n \pi$ and $V(x)=C \cos n \pi x, U(x)=$ $\left(-F(1) C /(n \pi)^{2}\right) \cos n \pi x$ where $C$ is an arbitrary constant. Hence the eigenspace belonging to $\left(\kappa_{n}\right)^{-1}=\left(\left(\theta+\lambda(n \pi)^{2}\right) / F(1)\right)^{-1}$ is generated by $\left(U_{n}, V_{n}\right), \quad U_{n}(x)=$ $-F(1) /(n \pi)^{2} \cdot \cos n \pi x, V_{n}(x)=\cos n \pi x$.

By Lemma 3.2, bifurcation does indeed occur for $\kappa=\kappa^{*}$, and we obtain a continuous branch of solutions of (3.6) all of which are nontrivial except for the solution $\left(\kappa^{*}, 0,0\right)$. A Lyapunov-Schmidt series expansion of these solution $(\kappa, U, V)$ near $\left(\kappa^{*}, 0,0\right)$ reveals that we have solutions of $(3.6)$ that correspond to the positive solutions of (2.1). In fact, let

$$
\begin{aligned}
& U(x)=\varepsilon \tilde{U}_{1}(x)+\varepsilon^{2} \tilde{U}_{2}(x)+\varepsilon^{3} \tilde{U}_{3}(x)+\cdots, \\
& V(x)=\varepsilon \tilde{V}_{1}(x)+\varepsilon^{2} \tilde{v}_{2}(x)+\varepsilon^{3} \tilde{v}_{3}(x)+\cdots, \\
& \kappa=\kappa^{*}+\tilde{\kappa}_{1} \varepsilon+\tilde{\kappa}_{2} \varepsilon^{2}+\cdots
\end{aligned}
$$


and we find that

$$
\begin{aligned}
& \tilde{U}_{1}^{\prime \prime}(x)=F(1) \tilde{V}_{1}(x), \\
& \tilde{V}_{1}^{\prime \prime}(x)=\tilde{V}_{1}(x)-\kappa^{*} F(1) \tilde{V}_{1}(x), \\
& \tilde{U}_{1}(1)=0, \quad \tilde{U}_{1}^{\prime}(0)=0, \quad \tilde{V}_{1}^{\prime}(0)=0, \quad \tilde{V}^{\prime}(1)=0 .
\end{aligned}
$$

Choose $\tilde{U}_{1}(x)=U_{1}(x), \tilde{V}_{1}(x)=V_{1}(x)$ and obviously $\tilde{\kappa}_{1}>0$ by Theorem 2.1(i).

To complete the proof for the existence part, we need to show that such a solution exists for all $\kappa>\kappa^{*}$. Since $\kappa^{*}$ is a simple characteristic value of $L^{*}$, it follows from Lemma 3.3 that there is a continuum $C_{\infty}^{+}$of solutions of (3.6) all of which are nontrivial except for the solution $\left(\kappa^{*}, 0,0\right)$ such that $C_{\infty}^{+}$either is unbounded or contains $\left(\kappa_{n}, 0,0\right)$ for some $n$.

Our approach is first to eliminate the latter possibility. Let $D_{+}^{\infty}$ be the nontrivial solutions of $(2.1)$ corresponding to $C_{+}^{\infty}$. We claim

$$
(\kappa, u, v) \in D_{\infty}^{+} \Rightarrow u>0, v>0 \text { and } \kappa \geq \kappa^{*} .
$$

Since $u>0, v>0$ near the bifurcation point $\left(\kappa^{*}, 1,0\right)$ and $C_{\infty}^{+}$is a continuum. If (3.10) does not hold then by Lemma 3.1 there exists $\left(\kappa_{0}, u, v\right) \in D_{\infty}^{+}$such that $u(0)=0$ or $v(0)=0$. If $u(0)=0$ then from $u^{\prime \prime}=F(u) v, u^{\prime}(0)=0$ it follows that $u \equiv 0$, which contradicts $u(1)=1$. On the other hand, if $v(0)=0$ then from $v^{\prime \prime}=-v\left(\kappa_{0} F(u)-\theta\right)$, $v^{\prime}(0)=0$ it follows that $v \equiv 0$ and hence $u \equiv 1$, which contradicts the fact $D_{+}^{\infty}$ does not contain a trivial solution. It is obvious from Lemma 3.1 that $\kappa \geq \kappa^{*}$.

Now we suppose $C_{\infty}^{+}$contains $\left(\kappa_{n}, 0,0\right)$ for some $n$. A Lyapunov-Schmidt expansion about $\left(\kappa_{n}, 0,0\right)$ as in (3.9) reveals

$$
\begin{aligned}
& U(x)=\varepsilon U_{n}(x)+\varepsilon^{2} \tilde{U}_{n, 2}(x)+\cdots, \\
& V(x)=\varepsilon V_{n}(x)+\varepsilon^{2} \tilde{V}_{n, 2}(x)+\cdots, \\
& \kappa=\kappa_{n}+\tilde{\kappa}_{n-1} \varepsilon+\cdots,
\end{aligned}
$$

where $U_{n}(x)=\left(-F(1) /(n \pi)^{2}\right) \cos n \pi x, V_{n}(x)=\cos n \pi x, \kappa_{n}=\left(\theta+\lambda(n \pi)^{2}\right) / F(1)$. It obviously contradicts (3.10) in a neighborhood of $\left(\kappa_{n}, 0,0\right)$. Hence $C_{\infty}^{+}$must be unbounded.

Now let $\Lambda, Y$ be the projections of $D_{+}^{\infty}$ onto the real axis and $B \times B$ respectively. To complete the proof of the existence part we show that

$$
\Lambda=\left[\kappa^{*}, \infty\right) .
$$

Suppose (3.11) does not hold. Then we may assume $\Lambda=\left[\kappa^{*}, \bar{\kappa}\right]$ and $Y$ is unbounded. Then there exists a sequence of points $\left\{\left(\bar{\kappa}_{n}, u_{n}, v_{n}\right)\right\}_{n=1}^{\infty}$ in $D_{+}^{\infty}$ such that $\bar{\kappa}_{n} \rightarrow \bar{\kappa}_{0} \in \Lambda$ and $\left\|\left(u_{n}, v_{n}\right)\right\| \rightarrow \infty$ as $n \rightarrow \infty$. Since $\left|u_{n}(x)\right| \leq 1$ for all $0 \leq x \leq 1$, by Lemma 3.1 and 3.4 it follows that $v_{n}(1) \rightarrow+\infty$ and $v_{n}(0) \rightarrow+\infty$ as $n \rightarrow \infty$. From Lemma 3.4, there exist $N_{0} \geq 0, C>0$ ( $C$ is independent of $n$ ) such that $v_{n}(0)>C v_{n}(1)$ for all $n \geq N_{0}$. Now we choose $\varepsilon>0$ sufficiently small that $\bar{\kappa} F(\varepsilon)-\theta<0$ and let

$$
x_{0}=\frac{C(\bar{\kappa} F(1)-\theta)}{C(\bar{\kappa} F(1)-\theta)-(\bar{\kappa} F(\varepsilon)-\theta)} ;
$$

then $0<x_{0}<1$. We claim:

There exists $n>N_{0}$ such that $u_{n}\left(x_{0}\right)<\varepsilon$. 
If (3.12) does not hold, then $u_{n}\left(x_{0}\right)>\varepsilon$ for all $n>N_{0}$ and hence $u_{n}(x) \geq \varepsilon$ for all $x_{0} \leq x \leq 1, n \geq N_{0}$. Then $u_{n}^{\prime \prime}(x) \geq F\left(u_{n}(x)\right) v_{n}(x) \geq F(\varepsilon) v_{n}(x) \geq F(\varepsilon) v_{n}(0)$ for all $x_{0} \leq x \leq 1$ and $\min _{x_{0} \leq x \leq 1} u_{n}^{\prime \prime}(x) \rightarrow+\infty$ as $n \rightarrow \infty$. But

$$
\begin{aligned}
u_{n}(1)-u_{n}\left(x_{0}\right) & =u_{n}^{\prime}\left(x_{0}\right) \cdot\left(1-x_{0}\right)+\frac{u_{n}^{\prime \prime}(\xi)}{2}\left(1-x_{0}\right)^{2} \\
& >\left\{\min _{x_{0} \leq x \leq 1} u_{n}^{\prime \prime}(x)\right\} \cdot\left(1-x_{0}^{2}\right) \rightarrow+\infty \text { as } n \rightarrow \infty,
\end{aligned}
$$

and this contradicts the fact that $0<u_{n}(1)-u_{n}\left(x_{0}\right)<1$. Hence we establish (3.12).

Consider $n$ as in (3.12). By the second equation in (2.1) we have

$$
\int_{0}^{1} v_{n}(x)\left[\kappa_{n} F\left(u_{n}(x)\right)-\theta\right] d x=0
$$

Let

L.H.S. of $(3.13)=\int_{0}^{x_{0}} v_{n}(x)\left[\kappa_{n} F\left(u_{n}(x)\right)-\theta\right] d x+\int_{x_{0}}^{1} v_{u}(x)\left[\kappa_{n} F\left(u_{n}(x)\right)-\theta\right] d x$.

Then

$$
\begin{aligned}
0 & <\int_{0}^{x_{0}} v_{n}(x)[\bar{\kappa} F(\varepsilon)-\theta] d x+\int_{x_{0}}^{1} v_{n}(x)\left[\kappa_{n} F(1)-\theta\right] d x \\
& <v_{n}(0)(\bar{\kappa} F(\varepsilon)-\theta) x_{0}+\left(1-x_{0}\right) v_{n}(1)(\bar{\kappa} F(1)-\theta) \\
& <v_{n}(0)(\bar{\kappa} F(\varepsilon)-\theta) x_{0}+C\left(1-x_{0}\right)(\bar{\kappa} F(1)-\theta) v_{n}(0) \\
& =v_{n}(0)\left[C(\bar{\kappa} F(1)-\theta)-x_{0}(C(\bar{\kappa} F(1)-\theta)-(\bar{\kappa} F(\varepsilon)-\theta))\right] \\
& =0 .
\end{aligned}
$$

Hence we obtain the desired contradiction and (3.11) holds. Q.E.D.

Our next step is to show the uniqueness of the nonnegative solution of (2.1), (2.2). Before we prove it, we present the following lemmas.

LEMMA 3.5. Let $\left(u_{1}, v_{1}\right),\left(u_{2}, v_{2}\right)$ be nonnegative solutions of (2.1) and (2.2) with $u_{1} \geq u_{2}$. Then $u_{1} \equiv u_{2}, v_{1} \equiv v_{2}$.

Proof. Suppose $u_{1} \geq u_{2}$ and $u_{1} Z u_{2}$. Let $\omega=v_{2} / v_{1}$. Then from (2.1), (2.2) we have

$$
\begin{aligned}
& \omega^{\prime \prime}+2\left(\frac{v_{1}^{\prime}}{v_{1}}\right) \omega^{\prime}+\omega\left[\kappa\left(F\left(u_{2}\right)-F\left(u_{1}\right)\right)\right]=0, \\
& \omega^{\prime}(0)=0, \quad \omega^{\prime}(1)=0 .
\end{aligned}
$$

Since $F\left(u_{2}\right)-F\left(u_{1}\right) \leq 0$, from the maximum principle [6] it follows that $\omega \equiv$ constant $>0$. But from (3.14) and $u_{1} \neq u_{2}$, we have a contradiction. Hence $u_{1} \equiv u_{2}$ and $v_{1} \equiv v_{2}$.

LEMMA 3.6. Let $\left(u_{1}, v_{1}\right),\left(u_{2}, v_{2}\right)$ be nonnegative solutions of $(2.1),(2.2)$ with $u_{1} \not u_{2}$. Then the curve $y=u_{1}(x)$ crosses the curve $y=u_{2}(x)$ a finite number of times on $0 \leq x \leq 1$.

Proof. From Lemma 3.5, the curve $y=u_{1}(x)$ must cross the curve $y=u_{2}(x)$ on $0 \leq x \leq 1$. Suppose $y=u_{1}(x)$ crosses the curve $y=u_{2}(x)$ an infinite number of times on $0 \leq x \leq 1$. Then there exists $\left\{x_{n}\right\}_{n=1}^{\infty}$ such that $u_{1}\left(x_{n}\right)=u_{2}\left(x_{n}\right)$ and there exists $a \in[0,1]$ such that $x_{n} \rightarrow a$ as $n \rightarrow \infty$. Obviously $u_{1}(a)=u_{2}(a)$. Let $U(x)=u_{1}(x)-u_{2}(x), 0 \leq x \leq 1$. Since for any neighborhood of $a$, the curve $y=u_{1}(x)$ crosses $y=u_{2}(x)$ an infinite 
number of times, the Taylor expansion of $U(x)$ at $a$ yields $U^{\prime}(a)=0, U^{\prime \prime}(a)=0$, $U^{\prime \prime \prime}(a)=0$. Hence $u_{1}^{\prime \prime \prime}(a)=u_{2}^{\prime}(a), u_{1}^{\prime \prime}(a)=u_{2}^{\prime \prime}(a), u_{1}^{\prime \prime \prime}(a)=u_{2}^{\prime \prime \prime}(a)$. From (2.1) we have $v_{1}(a)=v_{2}(a), v_{1}^{\prime}(a)=v_{2}^{\prime}(a), v_{1}^{\prime \prime}(a)=v_{2}^{\prime \prime}(a)$. However the uniqueness of the solution of the ordinary differential equations $(2.1)$ yields $u_{1} \equiv u_{2}, v_{1} \equiv v_{2}$. Hence we complete the proof of the lemma.

Proof of Theorem 2.1(ii) (uniqueness). Suppose we have two nonnegative solutions of (2.1), (2.1), say $\left(u_{1}, v_{1}\right),\left(u_{2}, v_{2}\right)$ with $u_{1} \neq u_{2}$ under the assumption $\kappa F(1)-\theta>0$. By Lemma 3.1(ii) $u_{1}, u_{2}, v_{1}, v_{2}$ are positive on $0 \leq x \leq 1$. From Lemmas 3.5, 3.6, the curve $y=u_{1}(x)$ crosses the curve $y=u_{2}(x)$ a finite number of times. Let $x_{0}=0, x_{n+1}=1$ and $x_{1}, \cdots, x_{n}$ be the points where two curves cross each other. Without loss of generality, we may assume $u_{1} \geq u_{2}$ on $\left[x_{k}, x_{k+1}\right]$, where $0 \leq k \leq n, k$ even, and $u_{2} \geq u_{1}$ on $\left[x_{k}, x_{k+1}\right]$ where $0 \leq k \leq n, k$ odd. In order to obtain a contradiction, we discuss two cases.

Case 1. $v_{1}(0) \leq v_{2}(0)$. Let $\omega=v_{2} / v_{1}$ on $0 \leq x \leq x_{1}$. Then we have

$$
\omega^{\prime \prime}+2\left(\frac{v_{1}^{\prime}}{v_{1}}\right) \omega^{\prime}+\omega\left[\kappa\left(F\left(u_{2}\right)-F\left(u_{1}\right)\right)\right]=0, \quad \omega^{\prime}(0)=0 .
$$

Then the maximum principle yields $v_{2}(x)>v_{1}(x)$ for all $0<x \leq x_{1}$. We claim $y=v_{2}(x)$ must cross $y=v_{1}(x)$ at some point $c_{1} \in\left(x_{1}, x_{2}\right)$. If not, then $v_{2} \geq v_{1}, u_{2} \geq u_{1}$ on $\left[x_{1}, x_{2}\right]$. Since $u_{2}\left(x_{2}\right)=u_{1}\left(x_{2}\right)$ and $u_{2}^{\prime}\left(x_{1}\right) \geq u_{1}^{\prime}\left(x_{1}\right), u_{2}\left(x_{1}\right)=u_{1}\left(x_{1}\right)$, it follows that

$$
\begin{aligned}
u_{1}\left(x_{2}\right)=u_{2}\left(x_{2}\right) & =u_{2}\left(x_{1}\right)+\left(x_{2}-x_{1}\right) u_{2}^{\prime}\left(x_{1}\right)+\int_{x_{1}}^{x_{2}} \int_{x_{1}}^{s} F\left(u_{2}(\eta)\right) v_{2}(\eta) d \eta d s \\
& >u_{1}\left(x_{1}\right)+\left(x_{2}-x_{1}\right) u_{1}^{\prime}\left(x_{1}\right)+\int_{x_{1}}^{x_{2}} \int_{x_{1}}^{s} F\left(u_{1}(\eta)\right) v_{1}(\eta) d \eta d s \\
& =u_{1}\left(x_{2}\right) .
\end{aligned}
$$

This is a contradiction. Similarly, let $\bar{\omega}=v_{1} / v_{2}$ on $c_{1} \leq x \leq x_{2}$. Then

$$
\bar{\omega}^{\prime \prime}+2\left(\frac{v_{2}^{\prime}}{v_{2}}\right) \bar{\omega}^{\prime}+\bar{\omega}\left[\kappa\left(F\left(u_{1}\right)-F\left(u_{2}\right)\right)\right]=0, \quad \bar{\omega}\left(c_{1}\right)=1 .
$$

The maximum principle yields $v_{1}>v_{2}$ on $\left(c_{1}, x_{2}\right]$.

Repeating the arguments shows that there exist $c_{2}, \cdots, c_{n}, x_{i}<c_{i}<x_{i+1}, i=1, \cdots, n$ such that $v_{1}\left(c_{i}\right)=v_{2}\left(c_{i}\right), i=1, \cdots, n, v_{1} \geq v_{2}$ on $\left[c_{i}, c_{i+1}\right]$ where $i$ is odd, and $v_{2} \geq v_{1}$ on $\left[c_{i}, c_{i+1}\right]$ where $i$ is even. If $u_{1} \geq u_{2}$ on $\left[x_{n}, 1\right]$ then $v_{2} \geq v_{1}$ on $\left[c_{n}, 1\right]$. Consider (3.15) on $\left[c_{n}, 1\right]$; then the maximum of $\omega=v_{2} / v_{1}$ occurs at $x=1$ but $\omega^{\prime}(1)=0$ and we obtain a contradiction. If $u_{2} \geq u_{1}$ on $\left[x_{n}, 1\right]$ then $v_{1} \geq v_{2}$ on [ $\left.c_{n}, 1\right]$. Similarly, consider (3.16) on $\left[c_{n}, 1\right]$; the maximum of $\bar{\omega}=v_{1} / v_{2}$ occurs at $x=1$, but $\bar{\omega}^{\prime}(1)=0$ and we obtain a contradiction.

Case 2. $v_{2}(0)<v_{1}(0)$. We claim that the curve $y=v_{1}(x)$ must cross $y=v_{2}(x)$ at some point $\bar{c}_{0} \in\left(0, x_{1}\right)$. If not, then $u_{1} \geq u_{2}, v_{1} \geq v_{2}$ on $\left[0, x_{1}\right]$. Since $u_{1}(0) \geq u_{2}(0), u_{1}^{\prime}(0)=$ $u_{2}^{\prime}(0)=0$, we have

$$
\begin{aligned}
u_{2}\left(x_{1}\right) & =u_{1}\left(x_{1}\right)=u_{1}(0)+\int_{0}^{x_{1}} \int_{0}^{s} F\left(u_{1}(\eta)\right) v_{1}(\eta) d \eta d s \\
& >u_{2}(0)+\int_{0}^{x_{1}} \int_{0}^{s} F\left(u_{2}(\eta)\right) v_{2}(\eta) d \eta d s=u_{2}\left(x_{1}\right)
\end{aligned}
$$


By the arguments in Case 1, there exist $\bar{c}_{1}, \cdots, \bar{c}_{n}$ such that $x_{i}<\bar{c}_{i}<x_{i+1}, i=1, \cdots, n$ such that $v_{1}\left(\bar{c}_{i}\right)=v_{2}\left(\bar{c}_{i}\right)$ and $v_{1}, v_{2}$ cross each other at $\bar{c}_{i}$. Applying the same arguments as in Case 1 we obtain a contradiction.

Hence we establish the uniqueness of solutions for (2.1), (2.2).

Discussion. We have established the existence and uniqueness of steady states for the equations (1.3), (1.4). As for the questions about the global behavior of solutions for this dynamical system, it is currently under investigation. From our numerical studies, the steady state should be globally asymptotically stable. This paper is the first step in discussing the effects of motility in the model studied in [1] which will provide a reasonable explanation for the phenomena in microbial ecology.

\section{REFERENCES}

[1] D. LAUfFenburger, R. ARIS AND K. Keller, Effects of random motility on growth of bacterial populations, Microb. Ecol., (1981), pp. 207-227.

[2] J. M. Cushing, Periodic time-dependent predator-prey systems, SIAM J. Appl. Math., 32 (1977), pp. 82-95.

[3] G. J. Butler AND H. I. Freldman, Periodic solutios of a predator-prey system with periodic coefficients, Math. Biosc., 55 (1981), pp. 27-38.

[4] M. A. KrasnoselskiI, Topological Methods in the Theory of Nonlinear Integral Equations. Macmillan, New York, 1964.

[5] P. H. RABINowitz, Some global results for nonlinear eigenvalue problems, J. Functional Analysis, 7 (1971), pp. 487-513.

[6] M. H. Protter and H. F. Weinberger, Maximum Principles in Differential Equations, Prentice-Hall, Englewood Cliffs, NJ, 1967. 